\title{
COMPARAÇÃO ENTRE PREDIÇÃO E PROJEÇÃO DA DISTRIBUIÇÃO DE DIÂMETROS DE POVOAMENTOS DE EUCALIPTO SUBMETIDOS A DESBASTES ${ }^{1}$
}

\author{
Helio Garcia Leite ${ }^{2}$, Aline Edwiges Mazon de Alcântara³, Daniel Henrique Breda Binot ${ }^{4}$, Ricardo \\ Rodrigues de Oliveira Neto ${ }^{4}$ e Mayra Luiz Marques da Silva ${ }^{6}$
}

\begin{abstract}
RESUMO - Dados de povoamentos de eucalipto submetidos a desbastes seletivos foram utilizados para modelagem da distribuição diamétrica. Foram ajustados dois modelos: um de predição e outro de projeção. Nos dois casos, o modelo de distribuição de diâmetros foi constituído por sistemas de equações não lineares. No primeiro caso, os parâmetros da função Weibull foram estimados em função de características dos povoamentos. No segundo, os parâmetros dessa função, observados em uma idade atual, foram empregados como variáveis independentes para serem estimados em idades futuras. Os resultados comprovaram maior eficiência na modelagem por meio de projeção.
\end{abstract}

Palavras-chave: Distribuição diamétrica, Predição, Projeção.

\section{COMPARISON BETWEEN PREDICTION AND PROJECTION OF THE DISTRIBUTION OF DIAMETERS OF EUCALYPT STANDS SUBMITTED TO THINNING}

\begin{abstract}
Data of eucalyptus stands submitted to selective thinning were used to diametric distribution modeling. Two models were adjusted: one for prediction and another for projection. In both cases, the model was constituted by non-linear equations systems. In the first case the parameters of the Weibull function were predicted in function of characteristics of the plantation. In the second, the parameters of that function, observed at the present age, were used as independent variables to estimate those parameters at further ages. The results proved a larger efficiency in the modelling through projection.
\end{abstract}

Keywords: Diametric distribution, Prediction, Projection.

\section{INTRODUÇÃO}

Um dos elementos essenciais do manejo florestal é a predição ou projeção do crescimento e da produção. Para este propósito são empregados modelos de crescimento e produção em nível de povoamento ou em nível de árvores individuais, ou de distribuição de diâmetros (CAMPOS; LEITE, 2009). Esta última categoria de modelos permite estimar crescimento e produção por classe de diâmetro, e sua principal característica é a presença de uma função densidade de probabilidade. Diferentes funções já foram empregadas, por exemplo: Beta (CLUTTER; BENNETT, 1965; PALAHI et al., 2007); Sb Johnson (HAFLEY; SCHUREUDER, 1977; SCOLFORO etal., 2003; PALAHÍ,

\footnotetext{
${ }^{1}$ Recebido em 25.02.2010 aceito para publicação em 11.03.2013.

${ }^{2}$ Departamento de Engenharia Florestal, Universidade Federal de Viçosa, UFV, Brasil. E-mail:<hgleite@ gmail.com>.

${ }^{3}$ CENIBRA - Celulose Nipo-Brasileira S/A . E-mail:<alimazon@ gmail.com>.

${ }^{4}$ Pesquisador Autônomo, DS. E-mail:<danielhbbinoti@gmail.com>.

${ }^{5}$ Floresteca.

${ }^{6}$ Universidade Federal do Vale do Jequitinhonha e Mucuri, MG, Brasil. E-mail:<mayrabinoti@ gmail.com>.
} 
2007) e Weibull (BAILEY ; DELL, 1973; MALTAMO et al., 2000; NOGUEIRA et al., 2001; BARRA et al., 2004; LEITEet al., 2005; ABBASI et al., 2008). Esta última função tem sido empregada com frequência em diferentes espécies e locais (CAMPOS; LEITE, 2009).

A estimação da distribuição de diâmetros pode ser feita empregando modelos de predição ou modelos de projeção. No primeiro caso, os parâmetros da distribuição estatística selecionada são correlacionados com parâmetros dos povoamentos (CLUTTER et al., 1983). No segundo, os parâmetros da distribuição estatística são estimados em função desses mesmos parâmetros observados em uma idade anterior (LEITE et al., 2005). Em qualquer caso, os modelos permitem estimar multiprodutos e simular desbastes (KNOBELL et al., 1986; CLUTTER et al., 1983; NOGUEIRA et al., 2005; SIIPILEHTO, 2007; CAMPOS; LEITE, 2009).

Diferentes enfoques têm sido empregados para modelar distribuições de diâmetros. No trabalho de Guimarães (1994), foi proposto um modelo de passo invariante, obtendo resultados compatíveis para a projeção da distribuição diamétrica a partir de quaisquer inventários iniciais conduzidos em povoamentos de eucalipto. Cao (2004) propôs dois métodos: de regressão de máxima verossimilhança e o da regressão da função de distribuição acumulativa. Nogueira et al. (2005) propuseram o emprego de um sistema de equações não lineares para projetar os parâmetros da função Weibull ajustada a dados de povoamentos de eucalipto submetidos a desbaste. Outras modificações, adaptações e aplicações podem ser encontradas em Burkhart (1971), Cao et al. (1982), Magnussen (1986), Gove e Patil (1998), Kangas e Maltamo (2000), Newton et al. (2005) e Mehtätalo et al. (2006).

Ao assumir que a forma e escala da distribuição de diâmetros, em uma idade qualquer, dependem da forma e escala observadas em uma idade anterior e que o tipo, a época de aplicação e o peso do desbaste afetam a forma e a escala, é factível admitir que os modelos de projeção possam ser mais eficientes do que os de predição.

Neste estudo foi avaliada a eficiência de um modelo de projeção em relação a um modelo de predição da distribuição de diâmetros de povoamentos de eucalipto submetidos a desbastes seletivos.

Revista Árvore, Viçosa-MG, v.37, n.2, p.321-328, 2013

\section{MATERIAL E MÉTODOS}

\subsection{Dados}

Os dados empregados neste estudo foram provenientes de um experimento sobre desbaste, instalado na região Nordeste do Estado da Bahia, Brasil, seguindo-se os princípios mencionados em Levels-of-Growing-Stock (2002).

O experimento foi localizado em três instalações, abrangendo locais de capacidade produtiva relativamente alta, média e baixa. Cada instalação consistiu de dois blocos, com duas repetições de cada tratamento em cada bloco, totalizando 48 parcelas experimentais de $2.600 \mathrm{~m}^{2}$ de área útil. O povoamento era composto de clones de um híbrido Eucalyptus grandis $\mathrm{x}$ Eucalyptus urophylla, plantado sob espaçamento inicial de 3,0 x 3,0 m. Os tratamentos aplicados corresponderam a 20\%,35\% e 50\% de área basal removida em cada desbaste, eliminando-se os piores indivíduos. Esse tipo de desbaste é conhecido como desbaste por baixo, pois as árvores colhidas pertencem às classes de copa inferiores. Este mesmo banco de dados foi utilizado nos dois tipos de modelos (predição e projeção). As parcelas permanentes foram mensuradas aos $27,40,50,58,61,76,87,101$ e 112 , $114,136,147,157$ e 165 meses, com desbastes realizados aos 58 e 147 meses. Em cada medição, foram medidos os diâmetros a 1,3 m de altura (dap) e a altura total de todas as árvores das parcelas.

\subsection{Distribuição estatística}

Para cada parcela em cada ocasião foi ajustada a função densidade de probabilidade Weibull (WEIBULL, 1951):

$$
f(X)=\left\{\frac{\gamma}{\beta}\left(\frac{(x-\alpha)}{\beta}\right)^{(\gamma-1)} e^{-\left(\frac{(x-\alpha)}{\beta}\right)^{\gamma}}\right.
$$

em que $\alpha$ é o parâmetro de locação, $\beta$ é $o$ parâmetro de escala $(\beta>0)$, $\gamma$ é o parâmetro de forma $(\gamma>0)$ e $\mathrm{x}$ é o centro de classe de diâmetro $(x>0)$.

Os dados de dap observados em cada parcela e idade foram agrupados em classes com amplitude de $1,0 \mathrm{~cm}$. O ajuste da função Weibull a esses dados foi feito pelo método de máxima verossimilhança, sendo o parâmetro $\alpha$ assumido como o diâmetro mínimo das árvores remanescentes. A aderência dos dados à distribuição Weibull foi avaliada pelo emprego do 
teste Kolmogorov-Smirnov (GIBBONS; SUBHABRATA, 1992; SOKAL; ROHLF, 1981) a $1 \%$ de probabilidade.

\subsection{Modelagem da distribuição de diâmetros}

O modelo de predição da distribuição diamétrica utilizado foi construído correlacionando características do povoamento com os parâmetros da função, sendo composto pelos seguintes modelos de regressão:

$$
\begin{aligned}
& d \max =e^{\left(\alpha_{0}+\frac{\alpha_{1}}{I}\right)}+\varepsilon \\
& \beta=e^{\left(\alpha_{0}+\alpha_{1} d \max +\frac{\alpha_{2}}{I}\right)}+\varepsilon \\
& \gamma=e^{\left(\alpha_{0}+\alpha_{1} \operatorname{Ln} \beta+\alpha_{2} d \min \right)}+\varepsilon \\
& d \min =e^{\left(\alpha_{0}+\alpha_{1} I\right)}+\varepsilon
\end{aligned}
$$

O modelo de projeção da distribuição diamétrica utilizado foi adaptado de Nogueira et al. (2005), sendo:

$$
\begin{aligned}
& \beta_{2}=\beta_{1}\left(\frac{I_{1}}{I_{2}}\right)+\alpha_{0}\left(1-\frac{I_{1}}{I_{2}}\right) d \max _{2}+\varepsilon \\
& L m \gamma_{2}=L n \gamma_{1} e^{\left(-\left(\alpha_{0}\right)\left(I_{2}{ }^{1}-I_{1}{ }^{1}\right)\right)_{+\varepsilon}^{\alpha}}{ }_{+\varepsilon}^{\alpha} \\
& d \max _{2}=d \max _{1}\left(\frac{I_{1}}{I_{2}}\right)+\alpha_{0}\left(1-\frac{I_{1}}{I_{2}}\right) \beta_{1}+\varepsilon \\
& d \min _{2}=d \min _{1} \mathrm{e}^{\alpha_{0}\left(I_{2}^{\alpha_{1}}-I_{1}^{\alpha_{1}}\right)}{ }_{+\varepsilon}
\end{aligned}
$$

em que $I_{1}$ e $I_{2}$ são as idades, atual e futura, em meses; $\gamma_{1}$ e $\gamma_{2}$ são os parâmetros de forma da função Weibull nas idades $I_{1}$ e $I_{2} ; \beta_{1}$ e $\beta_{2}$ são os parâmetros de escala da função Weibull nas $I_{1}$ e $I_{2} ; d \max _{1}$ e $\operatorname{dmax}_{2}$ são os diâmetros máximos $(\mathrm{cm})$ nas idades $I_{1}$ e $I_{2} ; d \min _{1}$ e $d m i n_{2}$ são os diâmetros mínimos $I_{1}$ e $I_{2} ; N_{1}$ e $N_{2}$ são as frequências de árvores por hectare nas idades $I_{1}$ e $I_{2}$; $L n$ indica o logaritmo neperiano; e $\alpha_{\mathrm{i}}$ são os parâmetros.

Os modelos foram ajustados pelos métodos de mínimos quadrados ordinários (modelos lineares) e de mínimos quadrados não lineares, pelo procedimento Quase Newton (modelos não lineares). O software utilizado foi o Statistica 8.0 (StatSoft, INC, 2009).

\subsection{Avaliação da redistribuição teórica}

A redistribuição teórica dos diâmetros foi avaliada pela capacidade dos sistemas de equações em predizer ou projetar os parâmetros da função Weibull ( $\beta$ e $\gamma$ ), bem como representar as intervenções realizadas. $\mathrm{O}$ coeficiente de correlação entre os valores observados e estimados dos parâmetros da função Weibull, juntamente com a análise gráfica dos resíduos, foi utilizado para avaliar a exatidão das estimativas de frequência por classe de diâmetro. Foram avaliados a consistência, a capacidade de projeção e o realismo biológico dos modelos, a partir de projeções de diferentes distribuições iniciais observadas. Para avaliar a exatidão das estimativas geradas pelas equações que compõem os modelos de distribuição de diâmetros, foram estimadas, ainda, as seguintes estatísticas: RMSE, Bias\% e desvio-padrão do erro de predição (SIIPILEHTO, 2000). Os estimadores empregados foram:

$\operatorname{RMSE}=\sqrt{\frac{\sum_{\mathrm{i}=1}^{\mathrm{n}\left(\mathrm{Y}_{\mathrm{i}}-\hat{\mathrm{Y}}_{\mathrm{i}}\right)^{2}}}{\mathrm{n}-1}} \quad$ bias $=\frac{1}{\mathrm{n}} \sum_{\mathrm{i}=1}^{\mathrm{n}}\left(\mathrm{Y}_{\mathrm{i}}-\hat{\mathrm{Y}}_{\mathrm{i}}\right)$

$\operatorname{bias}_{\%}=100 \frac{1}{n} \sum_{i=1}^{n}\left(Y_{i}-\hat{Y}_{i}\right) Y_{i} \quad S_{b}=\frac{1}{n} \sum_{i=1}^{n} \sqrt{\left(e_{i}-{\text { bias } \%)^{2}}^{2}\right.}$

em que:

$Y_{i}$ e $\hat{Y}_{i}=$ valores observados e estimados da variável sob análise;

$e_{i}=$ erro relativo de predição; e

$n=$ numero de casos.

\section{RESULTADOS}

Todos os ajustes da função Weibull resultaram em aderência pelo teste de Kolmogorov-Smirnorv (P>0,01), a $1 \%$ de significância. As distribuições estimadas para cada parcela e idade foram comparadas com as observadas, sendo o resultado das três parcelas selecionadas ao acaso, nas idades de 58, 112 e 147, apresentadas na Figura 1.

Com base nas análises gráficas dos resíduos (Figuras 2 e 3), foi observada uma distribuição aproximadamente normal dos resíduos ao aplicar as equações que compõem os modelos. Também pode ser observado o melhor desempenho dos modelos de projeção quando

Revista Árvore, Viçosa-MG, v.37, n.2, p.321-328, 2013 


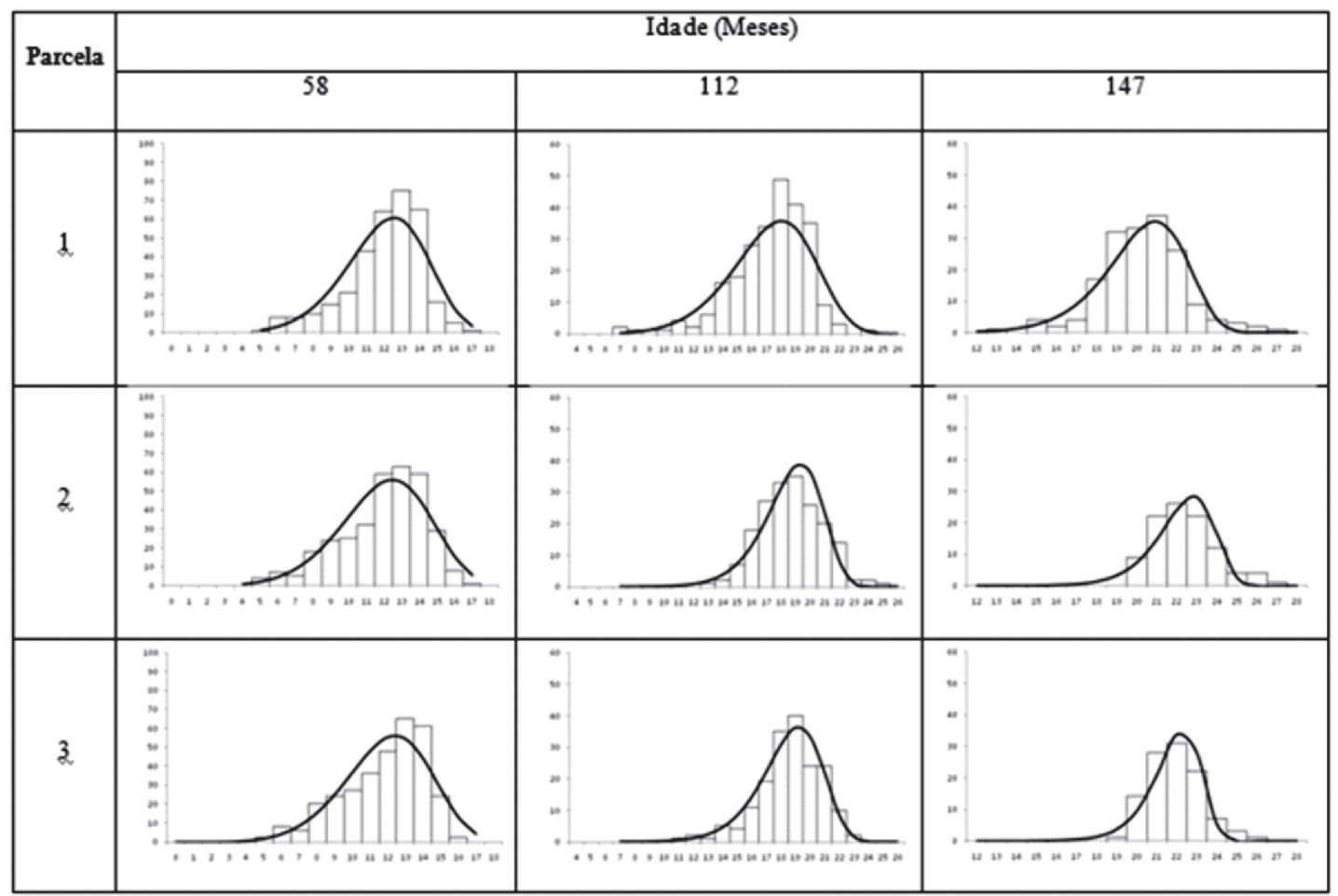

Figura 1 - Freqüências observadas e estimadas pelo modelo pela função Weibull para três parcelas selecionadas ao acaso, com intensidades de desbates de $35 \%$ para a parcela 1 e $50 \%$ para as parcelas 2 e 3.

Figure 1 - Frequencies observed and estimated by the Weibull function model for three randomly selected plots, with thinning intensities of $35 \%$ for plot 1 and $50 \%$ for plots 2 and 3 .

comparados com os modelos de predição. As estatísticas de precisão foram semelhantes àquelas obtidas na maioria dos estudos envolvendo modelagem de distribuição de diâmetros (Tabela 1).

As distribuições estimadas pelos dois tipos de modelos (predição e projeção), nas três parcelas escolhidas aleatoriamente, foram comparadas com as correspondentes distribuições observadas. Além disso, foram simulados dois desbastes, com redução de $35 \%$ da área basal remanescente (parcela 1) e 50\% (parcelas 2 e 3), nas idades de 58 e 147 meses. As distribuições estimadas aos 165 meses, pelos dois tipos de modelos, foram comparadas para avaliar a diferença na projeção dos modelos na idade de corte (Figura 4).

\section{DISCUSSÃO E CONCLUSÕES}

Diversas funções de densidade e probabilidade foram utilizadas para a construção de modelos de distribuição diamétrica, tendo destaque as funções
Beta e Weibull. A função Beta foi utilizada em uma primeira fase, começando com o trabalho de Clutter e Bennett (1965) e em vários outros, como Burkhart (1971). A partir da década de 1970, com o estudo de Bailey e Dell (1973) a função Weibull foi introduzida no setor florestal, predominando na maioria dos estudos de distribuição diamétrica (HAFLEY; SCHREUDER, 1977; MAGNUSSEN, 1986; LEITE, 1990; GUIMARÃES, 1994; SILVA, 2001; NOGUEIRA, 2001; NOGUEIRA et al., 2005; CAMPOS; LEITE, 2009).

A previsão da distribuição diamétrica é realizada, na maioria dos estudos, pela correlação dos parâmetros da função com características do povoamento, como diâmetros máximo, mínimo e médio e a idade, formando uma estrutura de predição (CAMPOS; TUMBULL, 1981; CAO et al., 1982; KNOEBELL et al., 1986; LEITE, 1990; KANGAS; MALTAMO, 2000; NEWTON et al., 2005; MEHTÄTALO et al., 2006; NORD-LARSEN; CAO, 2006). 
Comparação entre predição e projeção da distribuição de...

Tabela 1 - Modelos de predição e de projeção e suas estatísticas de ajuste.

Table 1 - Prediction and projection models and their adjustment statistics.

\begin{tabular}{|c|c|c|c|c|c|}
\hline Modelo de Predição: & $R_{\hat{y y}}^{2}$ & $R M S E$ & Bias & Bias\% & $S_{b}$ \\
\hline$d \min =e^{(1,1762+0,0109 I)}$ & 0,8683 & 0,7636 & 0,0026 & 0,0698 & 0,5342 \\
\hline$\gamma=e^{(2,3957-0,3450 \operatorname{Ln} \beta+0,0855 d \mathrm{~min})}$ & 0,9380 & 1,8392 & $-0,0370$ & 4,4710 & 4,5465 \\
\hline$d \max =e^{\left(3,4143-\frac{29,8178}{I}\right)}$ & 0,9445 & 2,7971 & 0,0734 & 2,9295 & 3,2542 \\
\hline$\beta=e^{\left(2,2015+0,0376 d \max -\frac{12,0076}{I}\right.}$ & 0,9853 & 1,5716 & 0,0323 & $-0,0779$ & 1,2344 \\
\hline \multicolumn{6}{|l|}{ Modelo de Projeção: } \\
\hline $\operatorname{dmin}_{2}=\operatorname{dmin}_{1} \mathrm{e}^{\left(-(31,4403)\left(I_{2}^{-0,01099}-I_{1}^{-0,01099}\right)\right)}$ & 0,8921 & 0,4594 & $-0,0153$ & 0,3436 & 0,4962 \\
\hline $\left.\operatorname{Ln} \gamma_{2}=\operatorname{Ln} \gamma_{1} e^{(-(313,5037)}\left(I_{2}^{0,00053}-I_{1}^{0,000536}\right)\right)$ & 0,8617 & 0,5827 & $-0,1495$ & 1,8203 & 1,9737 \\
\hline $\operatorname{dmax}_{2}=\operatorname{dmax}_{1}\left(\frac{I_{1}}{I_{2}}\right)+1,8911\left(1-\frac{I_{1}}{I_{2}}\right) \beta_{1}$ & 0,9773 & 0,8611 & $-0,0503$ & 1,4088 & 1,5533 \\
\hline$\beta_{2}=\beta_{1}\left(\frac{I_{1}}{I_{2}}\right)+1,1441\left(1-\frac{I_{1}}{I_{2}}\right) \mathrm{dmax}_{2}$ & 0,9875 & 1,8447 & 1,1665 & $-5,0514$ & 6,2180 \\
\hline
\end{tabular}
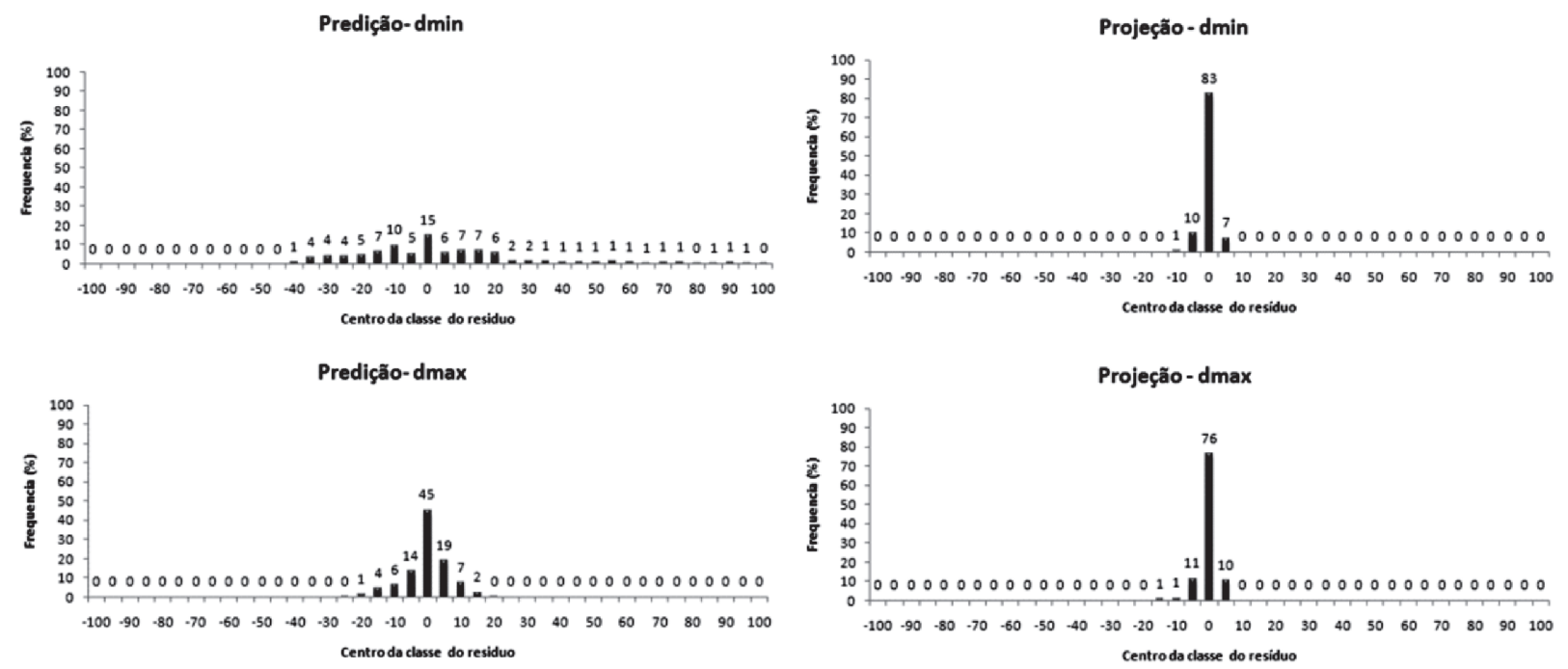

Figura 2 - Distribuições das classes de resíduo por freqüência, em porcentagem dos diâmetros mínimos e máximos, obtidas pela aplicação dos sistemas de equação que compõem os modelos de predição e projeção.

Figure 2 -Distributions of classes of residues by frequency in percentage of mininum and maximun diameters achieved by apllying the equation systems that constitute the prediction and projection models. 

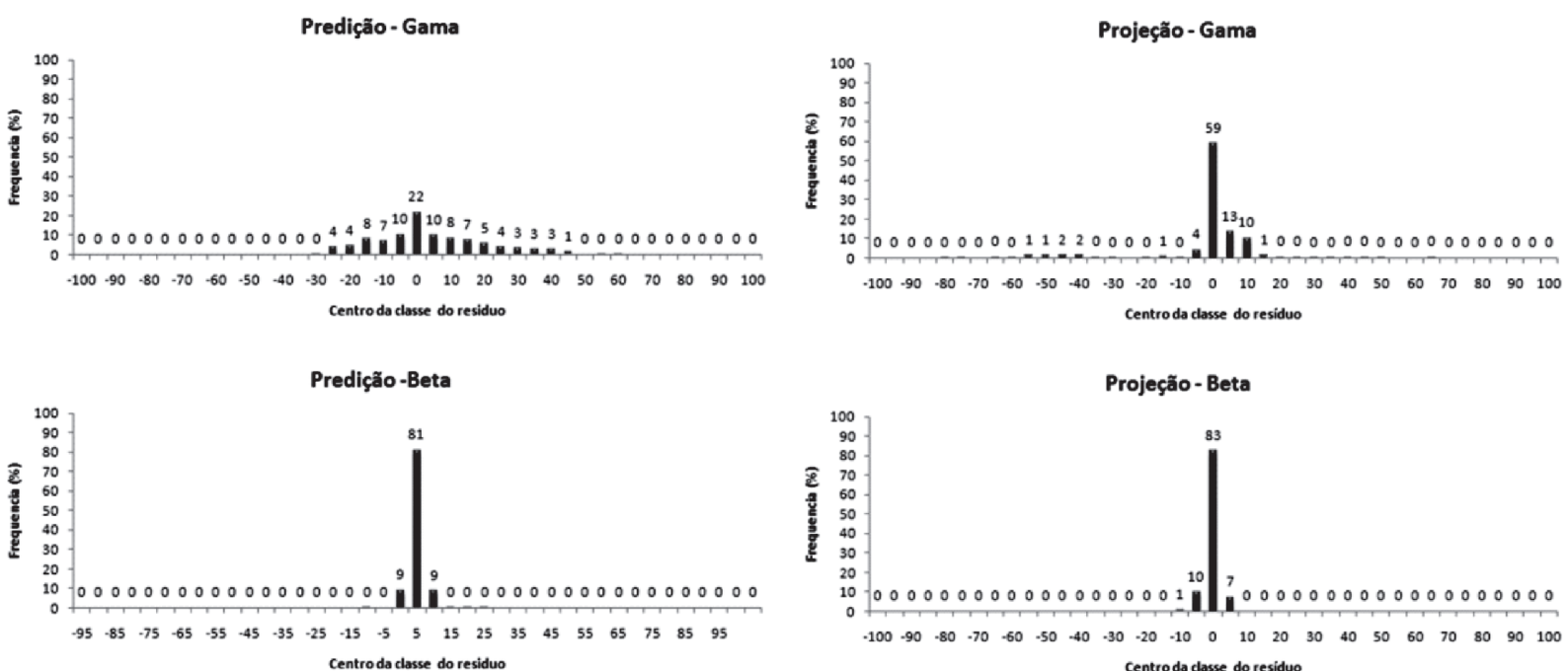

Figura 3 - Distribuições das classes de resíduo por freqüência, em porcentagem dos parâmetros beta e gama, obtidas pela aplicação dos sistemas de equação que compõem os modelos de predição e projeção.

Figure 3 -Distributions of classes of residues by frequency in percentage of beta and gama parameters achieved by using the equation systems that constitute the prediction and projection models.

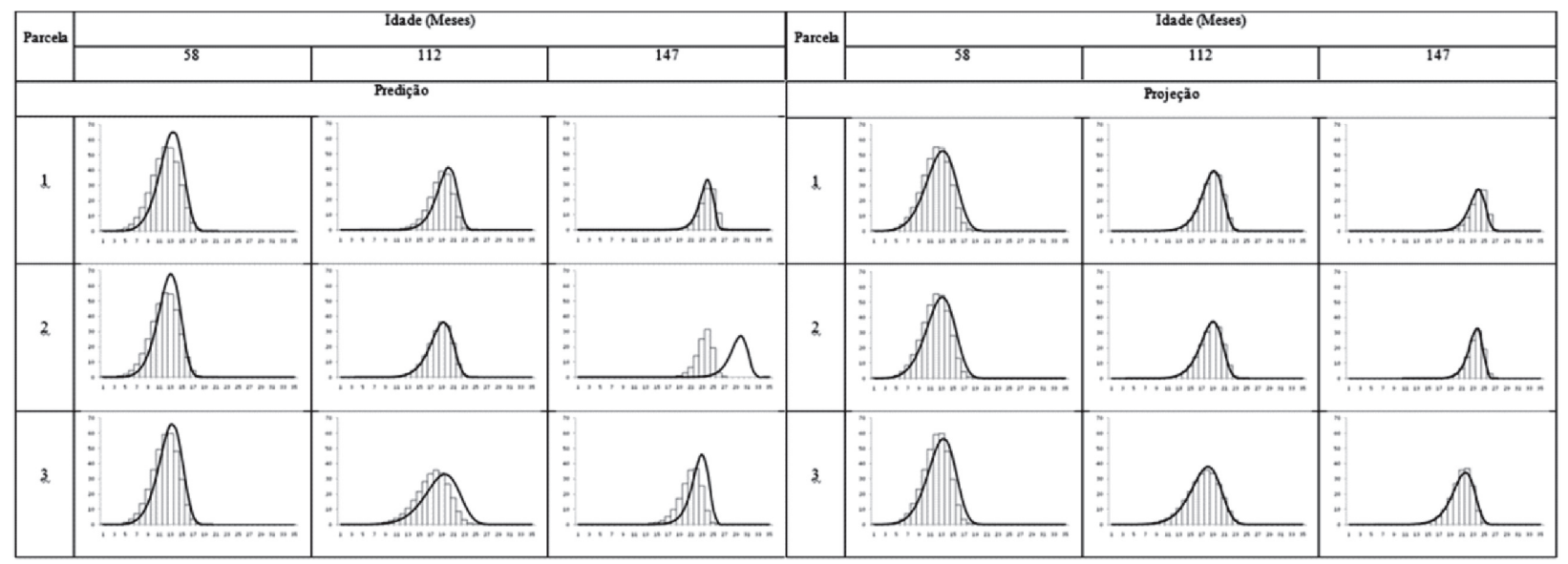

Figura 4 -Valores estimados pela função Weibull, e projetados pelos modelos de distribuição diamétrica para três parcelas escolhidas ao acaso, com intensidades desbaste de $35 \%$ para a parcela 1 e $50 \%$ para as parcelas 2 e 3 .

Figure 4 - Values estimated by the Weibull function and designed by diameter distribution models for three randomly chosen plots, with thinning intensities of $35 \%$ for plot 1 and $50 \%$ for plots 2 and 3 , respectively.

Modelos de distribuição diamétrica empregando as variáveis do povoamento em idades atual e futura, resultando em um sistema de projeção, foram propostos por Nogueira et al. (2005), sendo usados posteriormente por Leite et al. (2005), Lopes (2007) e Santos (2008), comprovando sua eficiência.

Revista Árvore, Viçosa-MG, v.37, n.2, p.321-328, 2013
O objetivo principal deste estudo foi avaliar a eficiência dos modelos de distribuições ajustados em uma estrutura de projeção em relação aos modelos no formato de predição. A melhoria na projeção dos parâmetros da função de distribuição diamétrica, em relação à predição, é comprovada pelos gráficos de resíduos apresentados

Revista Árvore, Viçosa-MG, v.37, n.2, p.321-328, 2013 
nas Figuras 2 e 3. Essa melhoria ocorreu devido à correlação significativa entre os parâmetros da função Weibull em idades sucessivas.

Mais diferenças entre frequências observadas e estimadas foram encontradas ao aplicar o modelo de predição, com superestimação nas maiores classes de dap (Figura 4). Erros nessas classes são mais prejudiciais ao manejo florestal, visto que as árvores dessas classes são mais importantes economicamente.

Ao analisar os resultados é possível inferir que os modelos de projeção são mais eficientes do que os modelos de predição. Isso fica bem claro na análise da Figura 3. Ao projetar a distribuição de diâmetros nos modelos de predição, observou-se tendência a superestimar os dados, o que não ocorreu nos modelos de projeção em nenhuma parcela analisada. Assim, recomendam-se incluir os parâmetros observados da função densidade de probabilidade empregada na modelagem, numa idade atual, como variáveis independentes nos modelos de regressão utilizados para projetar esses mesmos parâmetros, ou seja, para estimar uma variável futura ( variável $_{2}$ ) e utilizar no ajuste a mesma variável em idades anteriores (variável ${ }_{1}$ ). Os resultados permitiram concluir que a projeção da distribuição de diâmetros pode ser mais eficiente do que a usual predição.

\section{5 - REFERÊNCIAS}

ABBASI, B.; RABELO, L.; HOSSEINKOUCHACK, M. Estimating parameters of the three-parameter Weibull distribution using a neural network. European Journal of Industrial Engineering, v.2, n.4, p.428-445, 2008.

BAILEY, R. L., DELL, T. R. Quantifying diameter distributions with the Weibull function. Forest Science,v.19, n.2, p.97-104, 1973.

BARRA, O. S. V. et al.Proposta metodológica para o ajuste ótimo da distribuição diamétricaWeibull 3P.Floresta,v.34, n.3, p.387-393, 2004.

BURKHART, H.E. Slash pine plantations yields estimates based on diameter distribution: an evaluation. Forest Science, v.17,p.452-453, 1971.

CAMPOS, J. C. C.; LEITE, H. G.. Mensuração florestal: perguntas e respostas. 3.ed. Viçosa, MG: Universidade Federal de Viçosa, 2009.548p.
CAMPOS, J. C. C.; TURNBULL, K. J. Um sistema para estimar produção por classe de diâmetro e sua aplicação na interpretação do efeito de desbaste. RevistaÁrvore, v.5, n.1, p.1-16,1981.

CAO, Q. V. Predicting parameters of a Weibull function for modeling diameter distribution.Forest Science, v.50, n.4, p.682-685, 2004.

CAO, Q. V.; BURKHART, H. E.; LEMIN Jr., R. C. Diameter distributions and yields of thinned loblolly pine plantationsBlacksburg: School of Forestry and Wildlife Resources Virginia Polytechnic Institute and State University, 1982.(Publication, FWS-1-82)

CLUTTER, J.L.; BENNETT, F.A. Diameter distributions in old - field slash pine plantations.Georgia Forest Research Council Report, n.13, p.1-9, 1965.

CLUTTER, J.L. et al.Timber management: a quantitative approach. New York: John Willey \& Sons, 1983.333p.

GIBBONS, J.D.; SUBHABRATA, C.

Nonparametric statistical inference.3.ed. New York: Marcel Dekker, 1992. 544p. (Statistics: Textbook and Monograph, 31).

GOVE, J.H.; PATIL, G.P. Modelling basal áreasize distribution of forest stands: a compatible approach.Forest Science, v.44, n.2, p.285-297, 1998.

GUIMARÃES, D. P. Desenvolvimento de um modelo de distribuição diamétrica de passo invariante para prognose e projeção da estrutura de povoamentos de eucalipto.1994.178f. Tese (Doutorado em Ciência Florestal) - Universidade Federal de Viçosa, Viçosa, MG, 1994.

HAFLEY, W. L.; SCHREUDER, H. T. Statistical distributions for fitting diameter and height data in ever-aged stands.Canadian Journal of Forest Research,v.7, p.184-487, 1977.

KANGAS, A.; MALTAMO, M. Calibrating predicted diameter distribution with additional information. Forest Science,v.46, n.3, p.390-396,2000.

Revista Árvore, Viçosa-MG, v.37, n.2, p.321-328, 2013 
KNOEBELL, B. R.; BURKHART, H. E.; BECK, D. E. A growth and yield model for thinned stands of yellowpoplar. Forest Science, v.32, n.2, p.62, 1986.

LEITE, H. G. et al.Avaliação de um modelo de distribuição diamétrica ajustado para povoamentos de Eucalyptus sp. Submetidos a desbaste.Revista Árvore, v.29, n.2, p.271-280, 2005.

LEITE, H.G. Ajuste de um modelo de estimação de frequência e produção por classe de diâmetro, para povoamentos de Eucalyptussaligna Smith. 1990. 78f. Dissertação (Mestrado em Ciência Florestal) - Universidade Federal de Viçosa, Viçosa, MG, 1990.

LEVELS-OF-GROWING-STOCK. Cooperative study in Douglas-fir. Victoria, BC: Natural Resources Canada; Canadian Forest Service e Pacific Forestry, 2002. p.4.(InformationForestry)

LOPES, P.F. Modelo de distribuição de diâmetros para clones de eucalipto em sistema agroflorestal, 2007. 32f. Dissertação (Mestrado em Ciência Florestal) - Universidade Federal de Viçosa, Viçosa, 2007.

MAGNUSSEN, S. Diameter distribution inPiceaabies described by the weibull model. Scandinavian Journal Forest Science, n.1, p.493-502, 1986.

MALTAMO, M. et al. Comparison of percentile based prediction methods and the Weibull distribution in describing the diameter distribution of heterogeneous Scots pine stands.Forest Ecology and Management, v.133, n.3, p.263-274, 2000.

MEHTÄTALO, L.; MALTAMO, M.; KANGAS, A.The use of quantile trees in the prediction of the diameter distribution of a stand.Silva Fennica,v.40, p.3, p.501-516, 2006.

NEWTON, P.F.; ZHANG, S.Y.Stand-level diameter distribution yield model for black spruce plantations.Forest Ecologyand Management, v.209, p.181-192,2005.

NOGUEIRA, G. S. et al.Determinação da idade técnica de desbaste em plantações de eucalipto utilizando o método dos ingressos percentuais. ScientiaForestalis, n.59, p.51-59, 2001.

Revista Árvore, Viçosa-MG, v.37, n.2, p.321-328, 2013
NOGUEIRA, G. S.et al.Modelo de distribuição diamétrica para povoamentos de Eucalyptus sp. submetidos a desbaste. RevistaÁrvore, v.29, n.4, p.579-589, 2005.

NORD-LARSEN, T.; CAO, Q. V. A diameter distribution model for even-aged beech in Denmark.Forest Ecology and Management,v.231, p.218-225,2006.

PALAHÍ, M. et al.Comparison of beta, Johnson's SB, Weibull and truncated Weibull functions for modeling the diameter distribution of forest stands in Catalonia (north-east of Spain).European Journal of Forest Research, v.126, p.563-571, 2007.

SANTOS, F.L.Regulação da produção de floresta de eucalipto submetida a desbaste e destinada a multiprodutos. 2008. 92f. (Mestrado em Ciência Florestal) Universidade Federal de Viçosa, Viçosa, MG, 2008.

SCOLFORO, J. R. S.et al.Sb distribution accuracy to represent the diametric structure of Pinustaeda, through five methods. Forest Ecology and Management, v.175, n.1, p.489-496, 2003.

SIIPILEHTO, J. A comparison of two parameter prediction methods for stand structure in Finland.Silva Fennica, v.34, n.4 p.331-349, 2000.

SIIPILEHTO, J.; SARKKOLA, S.; MEHTÄTALO, L. Comparing regression estimation techniques when predicting diameter distributions of Scots pine on drained peatlands. Silva Fennica, v.41, n.2, p.333-349, 2007.

SILVA, A. A. L. Emprego de modelos de crescimento e produção em análise econômica de decisões de manejo florestal. 2001. 79f. Tese (Doutorado em Ciência Florestal) - Universidade Federal de Viçosa, Viçosa, MG, 2001.

SOKAL, R. R.; ROHLF, F. J. Biometry. San Francisco: Freeman, 1981. 859p.

STATSOFT, Inc. Statistica (data analysis software system), version 8.0, 2009.

WEIBULL, W. A statistical distribution function of wide applicability. Journal of Applied Mechanics, v.18, p.293-297, 1951. 